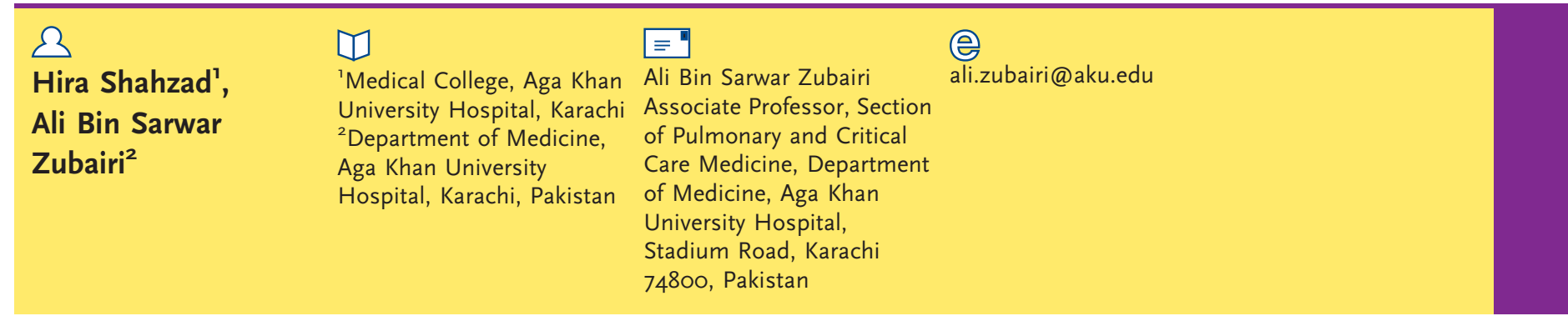

\title{
A 50-year-old woman with syncope
}

\section{Case report}

A 50-year-old postmenopausal woman with no prior comorbidity, was brought to the emergency department with two weeks history of exertional dyspnoea, syncope and generalised weakness. There was no associated chest pain or cough. She had experienced three episodes of syncope characterised by loss of consciousness, each episode lasting for $2-3$ mins.

On examination, she was a middle-aged woman with a pulse of 100 beats $\cdot \mathrm{min}^{-1}$, blood pressure of $120 / 80 \mathrm{mmHg}$, respiratory rate of 20 breaths $\cdot \mathrm{min}^{-1}$, room air oxygen saturation of $95 \%$ and jugular venous pressure within the normal range. Leg veins were prominent with minimal pedal oedema. Respiratory examination revealed an elliptical chest, normal vesicular breathing and occasional wheeze. Cardiovascular examination revealed a right ventricular heave, audible heart sounds with a systolic murmur audible at the tricuspid area and palpable peripheral pulses.

The chest radiograph (CXR) from a local hospital is shown in figure 1 .

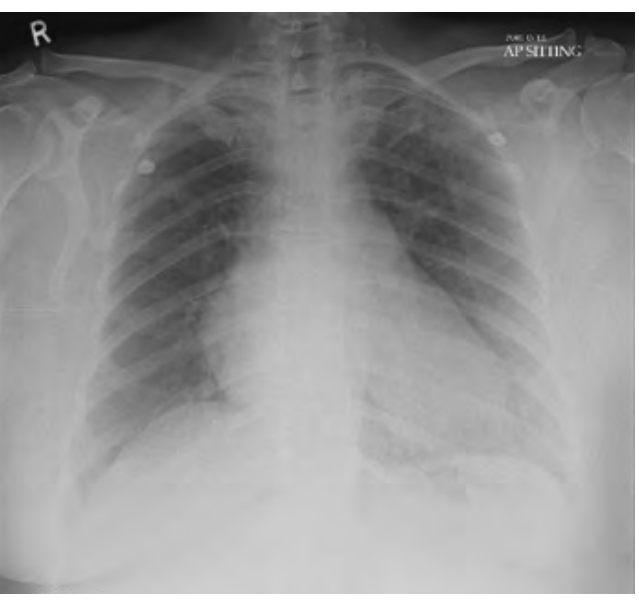

Figure 1

Chest radiograph.

\section{Task 1}

What does the chest radiograph demonstrate?
a. Left sided pleural effusion
b. Cardiomegaly
c. Lung infiltrates
d. Bilateral hilar prominence

Statement of interest None declared. 


\section{Answer 1}

b. Cardiomegaly

An electrocardiogram (EKG) revealed right ventricular strain with no evidence of an acute coronary episode. Initial investigations at our hospital showed a haemoglobin level of $14.9 \mathrm{mg} \cdot \mathrm{dL}^{-1}\left(11.5-13.8 \mathrm{mg} \cdot \mathrm{dL}^{-1}\right)$, total white cell count of $13.2 \times 10^{9}$ cells $\cdot L^{-1}\left(4.0-10.0 \times 10^{9}\right.$ cells $\left.\cdot L^{-1}\right)$ and platelets of $174 \times 10^{9}$ cells $\cdot L^{-1}$ $\left(150-400 \times 10^{9}\right.$ cells $\left.\cdot L^{-1}\right)$. Arterial blood gases showed $\mathrm{pH} 7.53$, oxygen tension 65.9, carbon dioxide tension 27.2, $\mathrm{HCO}_{3}^{-} 22.7$ and $\mathrm{O}_{2}$ saturation of $95.3 \%$.

Rest of the laboratory investigations including blood urea nitrogen, serum creatinine and electrolytes, pro-thrombin time were normal including an international normalised ratio (INR) of 1.08 and D-dimer levels of 1.914 .

A transthoracic echocardiogram (TTE) was obtained for evaluation of pulmonary hypertension. (fig. 2)

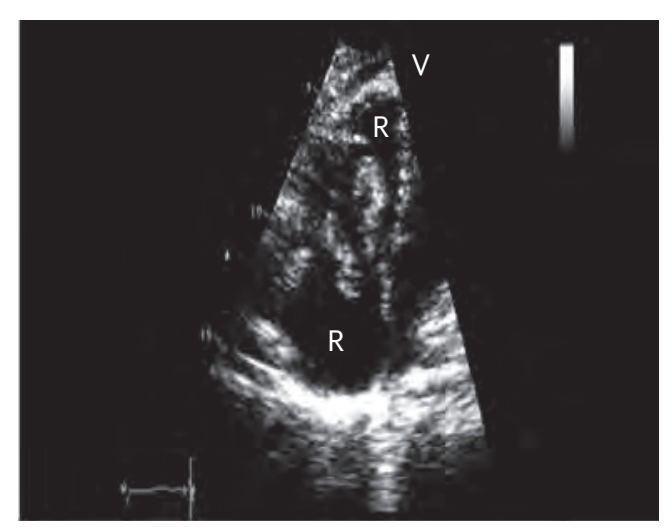

Figure 2

TTE.

\section{Task 2}

What does the TTE show in the right atrium?
a. Thrombi
b. Infective emboli
c. Cardiac mass
d. Metastatic lesion 


\section{Answer 2}

c. Cardiac mass.

TTE showed moderately dilated right atrium (RA) and ventricle (RV), severe tricuspid regurgitation, severely reduced RV function, normal left ventricular (LV) systolic function (ejection fraction of 55\%), pulmonary artery hypertension (PAH) of $90 \mathrm{mmHg}$ and a large, mobile multi-lobulated mass in RA protruding into $\mathrm{RV}$.

A spiral computed tomogram (CT) of the chest was obtained. (fig. 3)

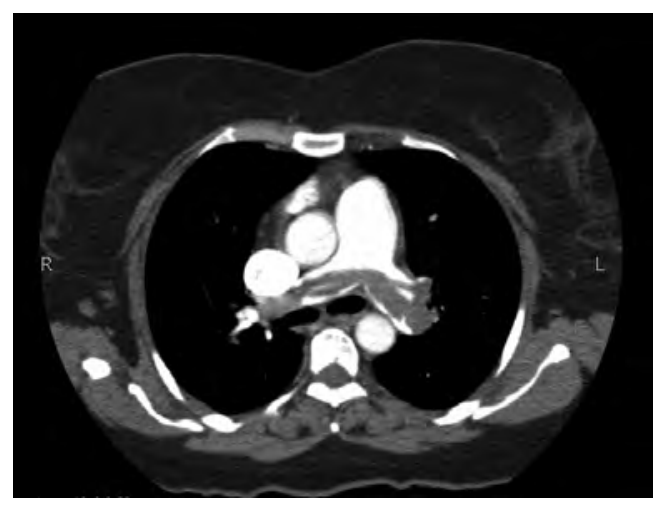

Figure 3

Spiral CT.

\section{Task 3}

What finding is evident from the spiral CT?

a. Opacities in the left lung zone

b. Right atrial mass

c. Embolus lodged in the pulmonary trunk

d. Foreign body in left main bronchus 
Answer 3

c. Embolus lodged in the pulmonary trunk

The CT of the chest revealed a saddle embolus residing in the main right and left PA with distal extension. The RA mass was not seen on CT. A diagnosis of massive pulmonary embolism (PE) with an atrial mass was made. The cardiothoracic surgery consultant deferred surgical removal of the mass due to high mortality risk. The patient underwent thrombolysis with tissue-plasminogen-activator (tPA) and was started on anticoagulation with subcutaneous enoxaparin at $80 \mathrm{mg}$ twice daily. The transoesophageal echocardiogram (TEE) on the third day of admission illustrated complete resolution of RA mass post-thrombolytic therapy, suggestive of a thrombus or a "clot in transit" (fig. 4). Enoxaparin was switched to warfarin with dose adjustment to maintain an INR between 2.0 and 3.0.

Further work-up was done in order to exclude hyper-coagulability secondary to an underlying malignancy. Patient was thus discharged on the eighth day of hospitalisation on adjusted warfarin anticoagulation therapy. Patient has been doing well since discharge 6 months ago.

\section{Discussion}

Presentation of a cardiac mass is a rare finding with an incidence of $0.001-0.28 \%$ reported in autopsy reports [1]. Differentials of such cardiac masses are commonly thrombi, primary cardiac tumors (most commonly atrial myxomas), metastatic tumors and infective

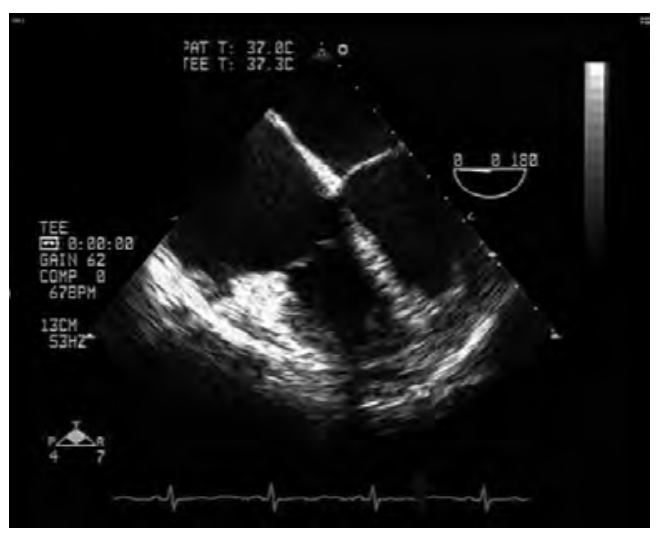

Figure 4

TEE on third day of admission. emboli. Acute thromboembolic disease has been increasingly associated with cardiac thrombi with an estimated incidence of 7$11 \%$ [2]. Due to the high risk of potential pulmonary and systemic embolisation, they require prompt therapeutic intervention. Once complication occurs in the form of pulmonary embolism (as in the present case), there is a threatened risk of compromised blood flow to the lungs and subsequently, right ventricular failure and an associated mortality rate of more than $15 \%$.

The distinction between an original atrial mass and a thrombus is not always easy. However, it is crucial since treatment is based on diagnosis; a tumour requires surgical resection, whereas a thrombus usually necessitates conservative treatment with immediate thrombolysis followed by anti-coagulation. Furthermore, an early diagnosis followed by rigorous treatment improves prognosis considerably.

Dyspnoea is the most common presenting symptom of pulmonary embolism. According to the International Cooperative Pulmonary Registry (ICOPER), $82 \%$ of the patients who were eventually diagnosed with PE had presented with dyspnoea [3]. Other symptoms include chest pain, cough and syncope. Patient-related predisposing factors include age 60 years and above, history of previous venous thromboembolism (VTE), malignancies, medical disorders causing prolonged bed rest and thrombophilia, hormone replacement therapy and oral contraceptive therapy. Almost 19\% of patients included in the ICOPER had no apparent predisposing risk factors for PE [3].

Treatment algorithms regarding massive and sub-massive pulmonary embolism have targeted the resolution of pre-existing clots using fibrinolytics (e.g. alteplase, streptokinase) in order to reduce the obstructive symptoms being caused by compromised pulmonary flow and cardiovascular function [4-6]. It also restores vascular integrity and prevents development of persistent pulmonary hypertension. Almost $92 \%$ of the patients recover within 36 hours both clinically and radiographically after institution of thrombolytic therapy [4]. Although it is known to work best if instituted within 48 hours of the onset of symptoms, it can still be employed 6-14 days after symptoms have appeared [4].

At the same time, an individual assessment of the patient's disease severity and the relative benefit versus risk evaluation is advised before thrombolysis is undertaken. 
According to the ACCP 2009 guidelines, "the decision to use thrombolytic therapy depends on the clinician's assessment of PE severity, prognosis and risk of bleeding". Fibrinolysis, once instituted, has the advantage of breakdown of preformed clots and restoration of cardiovascular and pulmonary blood flow. However, it also carries with itself the threat of potentially massive bleeding and intracranial haemorrhage $[4,5]$.

Short-term anticoagulation with intravenous or subcutaneous unfractionated heparin (UFH) or subcutaneous low molecular weight heparin (LMWH) in all patients with confirmed pulmonary embolism in the acute setting is recommended, where UFH is preferred over LMWH in massive and submassive acute $\mathrm{PE}$ and in individuals with renal impairment [6]. Patients should also receive long-term vitamin $\mathrm{K}$ antagonists ideally starting with heparin anticoagulation therapy on the first treatment day [6].

Almost $7-12 \%$ of the cases of VTE, where it occurs without any risk factors, have been associated with malignancies arising in the near future [4]. Patients who present with pulmonary embolism have a recurrence rate of $60 \%$ [4]. Moreover, the hypercoagulable malignant state predisposes to the metastatic spread of the tumour and anti-coagulants have evidently been reported to decrease the incidence of such progression. However, the use of extensive imaging techniques to screen for malignancies in all cases of VTE is controversial and is only advised in selective high-risk cases as assessed on the basis of careful history, examination and preliminary laboratory investigations.

\section{References}

1. McAllister HA Jr, Fenoglio || Jr. Tumors of the cardiovascular system. In: Atlas of tumor pathology. Washington: Armed Forces Institute of Pathology, 1978; pp. 1-20.

2. Chapoutot L, Nazeyrollas P, Metz D, et al. Floating right heart thrombi and pulmonary embolism: diagnosis, outcome and therapeutic management. Cardiology 1996; 87: 169-174.

3. Goldhaber SZ, Visani L, Rosa MD. Acute pulmonary embolism: clinical outcomes in the International Cooperative Pulmonary Embolism Registry (ICOPER). Lancet 1999; 353: 1386-1389.

4. Torbicki A, Perrier A, Konstantinides S, et al. Guidelines on the diagnosis and management of acute pulmonary embolism The Task Force for the Diagnosis and Management of Acute Pulmonary Embolism of the European Society of Cardiology (ESC). Eur Heart J 2008; 29: 2276-2315.

5. British Thoracic Society guidelines for the management of suspected acute pulmonary embolism. Thorax 2003; 58: 470-483.

6. Hirsh J, Guyatt G, Albers GW, et al. Executive summary: American College of Chest Physicians Evidence-Based Clinical Practice Guidelines (8th Edition). Chest 2008; 133: $71 \mathrm{~S}-109 \mathrm{~S}$. 